\title{
Ultrasound in emergency medicine: a colorful future in black and white
}

\author{
Anunaya R. Jain • Latha Stead · Wyatt Decker
}

Received: 20 October 2008 / Accepted: 21 October 2008 / Published online: 21 November 2008

(C) Springer-Verlag London Ltd 2008

From the experiment using an underwater bell to determine the speed of sound by J. D. Colladon in 1826, the generation of ultrasound waves by Francis Galton in 1876, the discovery of the piezoelectric effect by Pierre Curie in 1880, the development of sonar systems within a month of the sinking of the Titanic in 1912, to its early conception as the "hydrophone" by Paul Langevin and Constantin Chilowsky in 1915 and the development of medical ultrasonic systems since 1940, ultrasound is a technology that has now reached the "tipping point" and is being rapidly assimilated into multiple medical specialties beyond radiology.

Emergency medicine (EM) has witnessed the addition of ultrasonography to the arsenal of emergency care. The history of ultrasound in EM, spanning 15 years, can be traced back to the publication of the first emergency ultrasound curriculum by Mateer et al. in 1994. Increasing portability and the ease of training has fueled its rapid ascent to the front lines of EM practice to improve patient care.

Since the publication of the first formal policy statement for the use of ultrasound in EM by the American College of Emergency Physicians in 2001 [1], the concept of emer-

\footnotetext{
A. R. Jain

Department of Emergency Medicine, Mayo Clinic, Rochester, MN, USA

e-mail: jain.anunaya@mayo.edu

L. Stead $\cdot$ W. Decker $(\square)$

Emergency Medicine, College of Medicine, Mayo Clinic, Rochester, MN, USA

e-mail: decker.wyatt@mayo.edu

L. Stead

e-mail: stead.latha@mayo.edu
}

gency screening ultrasound has gained favor with most of the residency training programs in the USA and is now an element of the core curriculum required by the Residency Review Committee for Emergency Medicine [2]. It was also recently incorporated in the guidelines of the College of Emergency Medicine, UK in 2006 [3]. A large body of research studies has provided strong evidence that with appropriate training emergency ultrasound done by emergency physicians can safely aid time-critical decisions and procedures in the emergency department (ED).

The gamut of emergency ultrasound has gone from FAST (focused sonography for trauma) to comprehensive advanced scans and procedural guidance such as scans for shortness of breath, increased intracranial pressure (ICP), and central line placement. The ability of ultrasound to be incorporated in emergency medical services (EMS), telemedicine, and medical student education has mushroomed interest in the field. We are pleased to feature ultrasound in this issue of the International Journal of Emergency Medicine. The first of the ultrasound articles in this issue is a review on its use in EMS [4]. Not only does it highlight the current status of its use in Europe and the USA, but it also provides insight into the training programs, point of care use in resuscitation and trauma by EMS, and the potential application in mass casualty incident triage. There is still a long way to go as far as credentialing EMS personnel is concerned, because of the known learning curve in ultrasound applications.

The growth of ultrasound in EM is twofold - first is the development and validation of new point of care applications aptly demonstrated in the brief research report on "Ultrasound as an aid to reduction of pediatric forearm fractures" [5]. This application of ultrasound helps visually demonstrate successful reductions. Although the study did not demonstrate a decrease in the treatment time or 
radiological exposure, further validation will likely do so. The novelty of ultrasound applications has only multiplied with time, but the result remains the same: success.

The second prong of growth is the promulgation of basic training in developing systems of care as is exemplified in the study on "Introduction of a portable ultrasound unit into the health services of the Lugufu refugee camp, Kigoma District, Tanzania" [6]. The study demonstrates the feasibility and the ease with which ultrasound can be adapted to use in complex, underdeveloped care systems to enhance patient care and satisfaction. This article goes a step further to demonstrate that the initial expenditure for procuring the equipment is greatly offset by the time gain and the decreased stress on ancillary medical departments in developing systems.

The socioeconomic pressures of health care in all economies ranging from developed, developing and the underdeveloped will benefit from this boom of ultrasound in EM. But at the same time we must be wary that ultrasound in EM does not become a victim of its own success. Appropriate training and credentialing is the need of the hour.
The spiraling growth of ultrasound in EM has only one foreseen outcome, a future made colorful by black and white.

\section{References}

1. ACEP Policy Statement (2001) Emergency Ultrasound Guidelines. Approved ACEP Board of Directors

2. Heller MB, Mandavia D, Tayal VS et al (2002) Residency training in emergency ultrasound: fulfilling the mandate. Acad Emerg Med 9(8):835-839

3. Emergency medicine ultrasound - level 1 training document (2006) College of Emergency Medicine, Ultrasound Subcommittee. March

4. Nelson BP, Chason K (2008) Use of ultrasound by emergency medical services: a review. Int J Emerg Med doi:10.1007/s12245008-0075-6

5. Wong CE, Kee-Chong Ng, Angeline A (2008) Ultrasound as an aid to reduction of pediatric forearm fractures. Int $\mathrm{J}$ Emerg Med doi:10.1007/s12245-008-0072-9

6. Adler DH, Mgalula K, Price D, Taylor O (2008) Introduction of a portable ultrasound unit into the health services of the Lugufu refugee camp, Kigoma District, Tanzania. Int J Emerg Med doi:10.1007/s12245-008-0074-7 